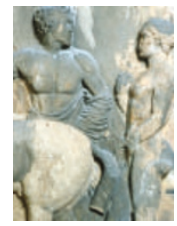

Culture clash

Lack of cash

threatens to hurt

Europe's heritage

p114

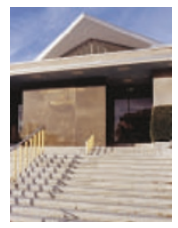

Free for all

US biomedical agency prepares for open publication p115

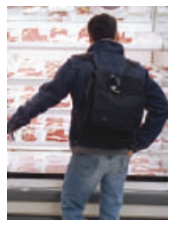

Bad taste

Consumer review falls foul of food chemist's ire p117

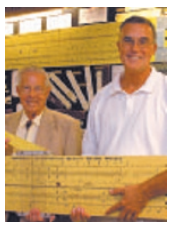

Logged on

Exhibition counts the contribution of slide rules

p119

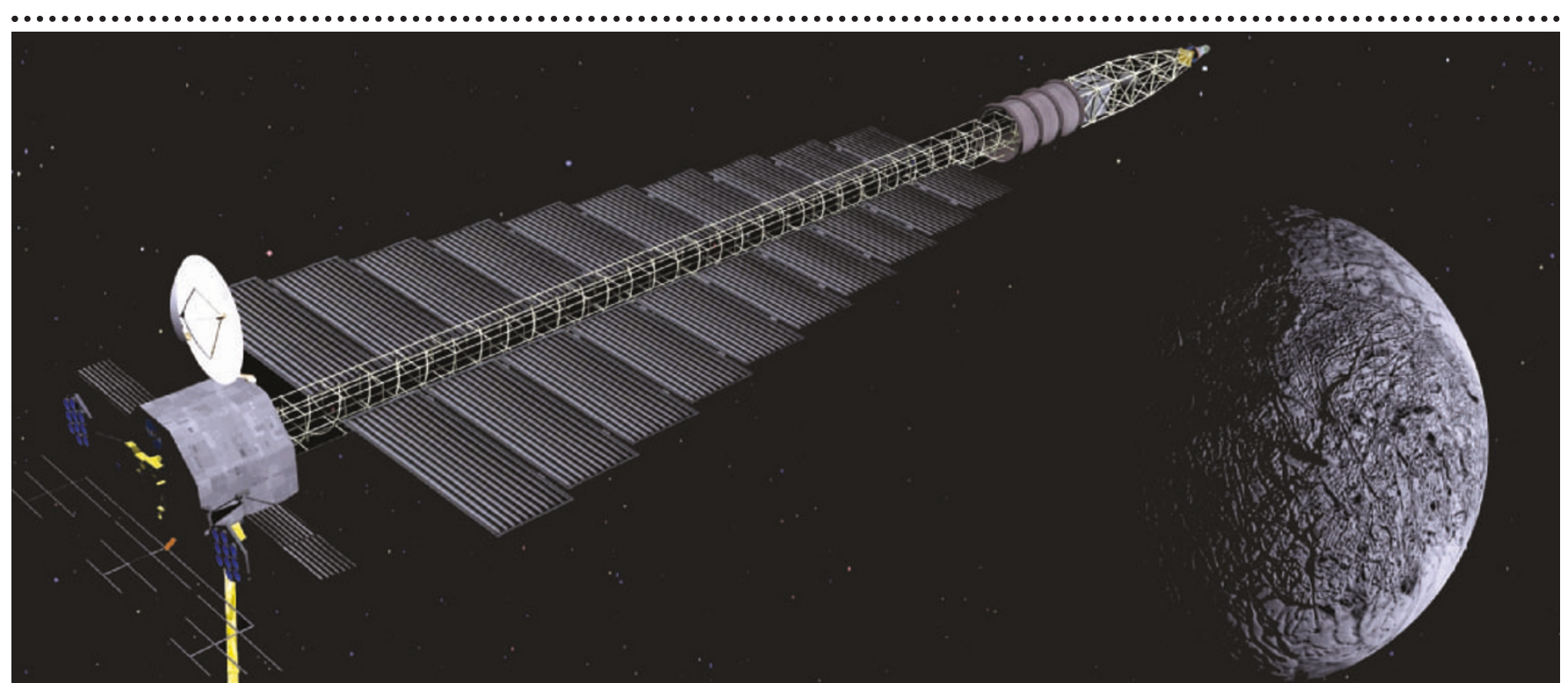

Up in the air: NASA is struggling to fund the development of the nuclear-powered craft proposed for the JIMO mission to Jupiter's moons.

\title{
Reviewers caution NASA over plans for nuclear-powered craft
}

\section{Tony Reichhardt, Washington}

An ambitious NASA project to use nuclear reactors to power spacecraft may be heading for trouble, based on early reactions in Congress and from scientists studying future space missions that would depend on them.

Later this month, NASA will choose an industrial contractor to begin designing its first such spacecraft, the proposed Jupiter Icy Moons Orbiter (JIMO). The mission, tentatively planned for launch around 2015, has strong personal backing from the agency's administrator Sean O'Keefe.

NASA surprised the scientific community last year by proposing JIMO, which would orbit several of Jupiter's moons at close range, and would be more technically capable than any scientific spacecraft ever built.

But many scientists are concerned about the mission's cost and political viability. NASA won't even hazard a guess on the final price tag until definition studies are completed next summer, says Ray Taylor, who heads Project Prometheus, the broader nuclear programme that includes JIMO. But estimates to build the nuclear propulsion system alone run as high as $\$ 4.5$ billion, and the spacecraft structure and science instruments would add billions more.
NASA officials claim that some of the upfront investment in designing JIMO will make future nuclear missions cheaper. But either way, the project promises to consume a significant fraction of the agency's current annual space science budget of $\$ 4$ billion.

JIMO would unquestionably be a big step up in technology. Its nuclear reactor would provide at least 10,000 watts of electricity for scientific instruments, against the 300 watts available to the Cassini spacecraft now orbiting Saturn. The power boost would allow sophisticated equipment such as icepenetrating radars to fly for the first time and would greatly increase the volume of data from cameras and other instruments.

But a National Academy of Sciences panel, asked by NASA to look at the value of nuclear power for space missions, met in Washington on 31 August to 2 September and seemed underwhelmed by presentations from NASA managers on possible follow-on missions to JIMO. A Neptune mission, for example, would still take nearly 20 years to return scientific data, offering no real advantage over conventional missions. And it would require much bigger rockets than the largest planned US launch vehicle to deliver the craft to Earth orbit before it headed into deep space.
Weighing the costs and long travel times against the scientific return, planetary scientist Reta Beebe of New Mexico State University in Las Cruces said at the meeting that the nuclear missions could be a hard sell with scientists. "This is not inspiring," she said.

Jerry Grey, a space-policy expert with the American Institute of Aeronautics and Astronautics who recently chaired a space nuclearpower round table in Washington, says that building JIMO poses no technical showstoppers, but "it ain't gonna be easy". It will require a thorough ground testing programme, and the risk of failure will still be high. Virtually everything on the mission will be new technology that will need to run for a decade or more without breaking down in space.

Daunting as these challenges are, JIMO is also off to a bad start politically. This summer the House appropriations committee cut $\$ 230$ million from next year's budget request of $\$ 438$ million for Project Prometheus. Unless the money is restored by the Senate, the effect on the programme would be "severe", says Taylor, who claims that Congress supports the project, and had to cut its funding because of competing budget demands. "To our knowledge, there's a tremendous amount of support" for it going ahead, he says. 